\title{
Developmental and metabolic brain alterations in rats exposed to bisphenol A during gestation and lactation
}

\author{
Nicolas Kunz ${ }^{\mathrm{a}, \mathrm{b}}$, Emily Jane Camm ${ }^{\mathrm{b}}$, Emmanuel Somm ${ }^{\mathrm{b}}$, Gregory Lodygensky ${ }^{\mathrm{b}}$, Stéphanie Darbre ${ }^{\mathrm{b}}$, \\ Michel Lucien Aubert ${ }^{b}$, Petra Susan Hüppi ${ }^{b}$, Stéphane Vladimir Sizonenko ${ }^{b}, *$, Rolf Gruetter ${ }^{\text {a,c,d }}$ \\ a Laboratory of Functional and Metabolic Imaging (LIFMET), Ecole Polytechnique Fédérale de Lausanne (EPFL), Lausanne, Switzerland \\ ${ }^{\mathrm{b}}$ Division of Child Development $\mathcal{E}$ Growth, Department of Pediatrics, University of Geneva, Geneva, Switzerland \\ ${ }^{c}$ Department of Radiology, University of Lausanne, Lausanne, Switzerland \\ d Department of Radiology, University of Geneva, Geneva, Switzerland
}

\section{A R T I C L E I N F O}

\section{Article history:}

Received 8 July 2010

Received in revised form

14 September 2010

Accepted 27 September 2010

\section{Keywords:}

Bisphenol A

Brain development

Cerebral metabolism

Nuclear magnetic resonance

Malate-aspartate shuttle

Mitochondria

\begin{abstract}
A B S T R A C T
In recent years, considerable research has focused on the biological effect of endocrine-disrupting chemicals. Bisphenol A (BPA) has been implicated as an endocrine-disrupting chemical (EDC) due to its ability to mimic the action of endogenous estrogenic hormones.

The aim of this study was to assess the effect of perinatal exposure to BPA on cerebral structural development and metabolism after birth.

BPA $(1 \mathrm{mg} / \mathrm{l})$ was administered in the drinking water of pregnant dams from day 6 of gestation until pup weaning. At postnatal day 20 , in vivo metabolite concentrations in the rat pup hippocampus were measured using high field proton magnetic resonance spectroscopy. Further, brain was assessed histologically for growth, gross morphology, glial and neuronal development and extent of myelination.

Localized proton magnetic resonance spectroscopy $\left({ }^{1} \mathrm{H}\right.$ MRS) showed in the BPA-exposed rat a significant increase in glutamate concentration in the hippocampus as well as in the Glu/Asp ratio. Interestingly these two metabolites are metabolically linked together in the malate-aspartate metabolic shuttle.

Quantitative histological analysis revealed that the density of NeuN-positive neurons in the hippocampus was decreased in the BPA-treated offspring when compared to controls. Conversely, the density of GFAP-positive astrocytes in the cingulum was increased in BPA-treated offspring.

In conclusion, exposure to low-dose BPA during gestation and lactation leads to significant changes in the Glu/Asp ratio in the hippocampus, which may reflect impaired mitochondrial function and also result in neuronal and glial developmental alterations.
\end{abstract}

(C) 2010 ISDN. Published by Elsevier Ltd. All rights reserved.

\section{Introduction}

In recent years, considerable research has focused on the association between exposure to endocrine-disrupting chemicals and the onset of disease and reproductive impairment. BPA $\left(4,4^{\prime}-\right.$ isopropylidenediphenol), which is widely used in plastic food and drink containers, has been implicated as an endocrine-disrupting chemical due to its ability to mimic the action of endogenous estrogenic hormones (Paris et al., 2002; Ranjit et al., 2009; Vom Saal et al., 1998).

Several studies have confirmed the presence of BPA in urine in human populations $(0.3-7.9 \mu \mathrm{g} /$ day) (Calafat et al., 2005;

\footnotetext{
* Corresponding author at: Service du Développement et de la Croissance, Département Enfant \& Adolescent, Hôpital des Enfants, 6 rue Willy Donzé, 1211 Geneva 14, Switzerland. Tel.: +4122382 54 93; fax: +41 223824315 .

E-mail address: stephane.sizonenko@unige.ch (S.V. Sizonenko).
}

Fujimaki et al., 2004; Takeuchi and Tsutsumi, 2002). BPA has been detected in the serum of pregnant women $(1-2 \mathrm{ng} / \mathrm{ml})$, fetus $\operatorname{serum}(0.2-9.2 \mathrm{ng} / \mathrm{ml})$, amniotic fluid $(8.3-8.7 \mathrm{ng} / \mathrm{ml})$, placental tissue (1.0-104.9 ng/g), and breast milk (1.1 ng/l) (Ikezuki et al., 2002; Schonfelder et al., 2002; Ye et al., 2005), suggesting that the human fetus is readily exposed to this compound during pre- and postnatal development.

Furthermore, a number of studies have shown behavioural modifications after exposure to BPA during gestation and/or lactation at low and high doses (i.e. $40-400 \mu \mathrm{g} / \mathrm{kg} /$ day), such as masculinisation behaviour in female pups or hyperactivity (Dessi-Fulgheri et al., 2002; Farabollini et al., 2002; Ishido et al., 2004; Palanza et al., 2008). These behavioural changes suggest some effects of BPA on the developing central nervous system (CNS). The effect of BPA on the CNS may be of increased importance in the fetal and postnatal brain development and appears now as a public health concern (Vandenberg et al., 2009). To date, there have been few in vivo studies that have examined the impact of BPA exposure during 
Table 1

Primary associated role of the metabolites quantified by ${ }^{1} \mathrm{H}$ MRS and abbreviation in this study.

\begin{tabular}{|c|c|c|}
\hline Primary associated role & Compound & Abbreviation \\
\hline Antioxidants & $\begin{array}{l}\text { Ascorbate } \\
\text { Glutathione }\end{array}$ & $\begin{array}{l}\text { Asc } \\
\text { GSH }\end{array}$ \\
\hline Excitatory amino acid & Aspartate & Asp \\
\hline Excitatory neurotransmitter & $\begin{array}{l}\text { Glutamate } \\
\text { N-Acetylaspartylglutamate }\end{array}$ & $\begin{array}{l}\text { Glu } \\
\text { NAAG }\end{array}$ \\
\hline $\begin{array}{l}\text { Precursor of Glu located in astrocytes } \\
\text { Inhibitory neurotransmitter }\end{array}$ & $\begin{array}{l}\text { Glutamine } \\
\gamma \text {-Aminobutyric acid }\end{array}$ & $\begin{array}{l}\text { Gln } \\
\text { GABA }\end{array}$ \\
\hline Membrane synthesis & $\begin{array}{l}\text { Glycerophosphorylcholine } \\
\text { Phosphorylcholine } \\
\text { Phosphorylethanolamine }\end{array}$ & $\begin{array}{l}\text { GPC } \\
\text { PCho } \\
\text { PE }\end{array}$ \\
\hline $\begin{array}{l}\text { Source of energy } \\
\text { End product of anaerobic glycolysis }\end{array}$ & $\begin{array}{l}\text { D-Glucose } \\
\text { Lactate }\end{array}$ & $\begin{array}{l}\text { Glc } \\
\text { Lac }\end{array}$ \\
\hline Reservoir of generation of ATP & $\begin{array}{l}\text { Creatine } \\
\text { Phosphocreatine }\end{array}$ & $\begin{array}{l}\mathrm{Cr} \\
\mathrm{PCr}\end{array}$ \\
\hline Putative neuronal marker & $\mathrm{N}$-Acetyl-aspartate & NAA \\
\hline Osmoregulation & $\begin{array}{l}\text { Taurine } \\
\text { Myo-inositol }\end{array}$ & $\begin{array}{l}\text { Tau } \\
\text { Ins }\end{array}$ \\
\hline
\end{tabular}

gestation and lactation on brain development (Facciolo et al., 2002). In addition, BPA (25-50 mg/kg/day, i.p. injection) has been shown to enhance oxidative stress and lipid peroxidation promoting the cellular death in several organs (e.g. brain, liver, and kidney) of exposed rodents (Aydogan et al., 2008; Kabuto et al., 2003).

Some investigators have reported the beneficial effects of BPA on brain development, mediated for the most part by estrogen receptors (ERs) (Kubo et al., 2004; Shikimi et al., 2004) (50-200 $\mu \mathrm{M}$ of BPA in cell culture or injection of $500 \mu \mathrm{g} /$ day during 5 days in the rat cerebrospinal fluid, respectively). But most studies, however, have shown the potential risk of BPA exposure on brain development. In vitro, BPA exposure has been shown to inhibit oligodendrocyte precursor cell differentiation and myelin basic protein (MBP) expression (Seiwa et al., 2004), and to influence synaptogenesis and neuronal vulnerability in hippocampal cultures (Sato et al., 2002). In vivo, BPA inhibits estrogen-activated hippocampal spine synapse formation (Maclusky et al., 2005; Zhou et al., 2009) (subcutaneous injection of $40-400 \mu \mathrm{g} / \mathrm{kg}$ of BPA in the rat and from gestational day (GD) 8 to PND21 $20 \mu \mathrm{g} / \mathrm{kg} /$ day of BPA, respectively) and disrupts normal neocortical development by accelerating neuronal differentiation/migration (Nakamura et al., 2006) (subcutaneous injection of $20 \mu \mathrm{g} / \mathrm{kg} / \mathrm{day}$ from GD0). It is therefore of substantial interest to investigate the effects of pre and post-natal exposures of BPA on brain development. In particular, in view of the potential translation to human studies, the approach through non-invasive techniques is attractive. ${ }^{1} \mathrm{H}$ MRS is an in vivo investigational approach that has been used to study the metabolic changes during rat brain development (Tkac et al., 1999). This technique allows the assessment of the concentration of 21 metabolites, termed "neurochemical profile" (Pfeuffer et al., 1999) (cf. Table 1). Previous studies have shown that impaired nutrient supply, such as iron deficiency (Rao et al., 2003) or chronic hypoxia (Raman et al., 2005) leads to specific changes in the neurochemical profile that implicate delayed myelination and impaired development of the neuropil.

The aim of the current study was to assess the effects of BPA exposure during gestation and lactation on cerebral metabolism and development by using in vivo localized ${ }^{1} \mathrm{H}$ MRS in combination with quantitative histopathology.

\section{Materials and methods}

\subsection{Animals}

All animal procedures were reviewed and approved by the university and state animal ethics boards. Virgin female (275-300 g) and male (300-325 g)
Sprague-Dawley (Taconic Europe, The Netherlands) rats were used for breeding Pregnant dams were housed individually under standard conditions (12 h:12 h light-dark cycle). To avoid BPA induced potential malformation during the first days of gestation (Nakamura et al., 2006; Xing et al., 2010), the administration of BPA started from day 6 of gestation through postnatal day 20 (PND20) of lactation, where dams were given $1 \mathrm{mg} / \mathrm{l} \mathrm{BPA}$ (dissolved in 1\% ethanol) in their drinking water (Rubin et al., 2001; Somm et al., 2009). The mean level dosage of intake of the dams was estimated to approximately $70 \mu \mathrm{g} / \mathrm{kg} /$ day by weighting the drinking bottle every day. This dose is in the range that does induce neural and behavioura changes. The dams of control group received drinking water containing the same amount of ethanol (1\%) without BPA. The end of the treatment coincided with the weaning age (PND20).

To control for dietary phytoestrogen intake, control and BPA-treated dams were given a chow diet low in phytoestrogens, with genistein content below the detection limit (Kliba Nafag 3250, Provimi Kliba, Kaiseraugst, Switzerland) 10 days prior to mating and throughout the gestation and lactation. BPA-free cages and drinking bottles were used to avoid contamination. Male and female data were combined for all analyses since no gender differences between BPA-treated and control-offspring's neurochemical profiles were seen. Furthermore, the number of offspring per litter and the ratio of male to female offspring at birth were not statistically different between control and BPA-treated rats.

\subsection{Nuclear magnetic resonance (NMR)}

Localized ${ }^{1} \mathrm{H}$ MRS was focused on the investigation of metabolic changes in the hippocampus region since several studies have reported neuronal alterations in hippocampal cultures after BPA exposure (Choi et al., 2007; Lee et al., 2008; Maclusky et al., 2005; Ogiue-Ikeda et al., 2008; Sato et al., 2002; Zhou et al., 2009). Pups from the two animal groups ( $n=8$ BPA-treated group, $n=6$ control), were studied at PND20. Pups were anesthetized using a mix of isoflurane (3\%) and oxygen, and then maintained under anesthesia using a nose cone with $1-1.5 \%$ of isoflurane during the MRS acquisition (on average $90 \mathrm{~min}$ ). During the MRS experiment pups were maintained in a specific home-built holder to restrain head motion. The body temperature was maintained at $37.5 \pm 1{ }^{\circ} \mathrm{C}$ by circulating heated water and breath rate was monitored during the whole experiment. To control for basic physiological status of the animal, whole blood lactate measurements were done before and after the MRS acquisition on a GM7 Micro-Stat (Analox Instruments Ltd., UK) by tail-bleeding; no difference in blood lactate was observed.

\subsubsection{Proton spectroscopy}

All ${ }^{1} \mathrm{H}$ MRS examinations were performed on a Varian INOVA console (Varian, Palo Alto, CA) connected to an actively shielded $9.4 \mathrm{~T} / 31 \mathrm{~cm}$ magnet (Magnex Scientific, Abingdon, UK) with actively shielded gradients $(400 \mathrm{mT} / \mathrm{m}$ in $120 \mu \mathrm{s}, 12 \mathrm{~cm}$ inner diameter). A home-built $17 \mathrm{~mm}$-diameter ${ }^{1} \mathrm{H}$ quadrature surface coil was used for radio frequency transmission and signal reception. A fast spin echo magnetic resonance imaging (MRI) sequence (echo train length $=16$, echo spacing $=10 \mathrm{~ms}$, effective echo time $=80 \mathrm{~ms}$ ) was used to obtain anatomical images with an in plane resolution of $\sim 80 \mu \mathrm{m}$ and a slice thickness of $0.8 \mathrm{~mm}$. Based on these images, the volume of interest (VOI) $(2 \mathrm{~mm} \times 3 \mathrm{~mm} \times 2 \mathrm{~mm})$ for spectroscopy was positioned on the left hippocampus. An ultra-short echo time $(\mathrm{TE}=2 \mathrm{~ms})$ localized STEAM-based sequence was used for in vivo ${ }^{1} \mathrm{H}$ MRS. The STEAM sequence is based on three 90 radio frequency pulses affording a robust spatial localization and allows short echo time measurements (Tkac et al., 1999). The water signal was suppressed by a VAPOR 
scheme integrating three outer volume suppression modules (Tkac et al., 1999). First and second order shim current were adjusted with an EPI-based FASTMAP sequence (Gruetter, 1993) on a volume of $3 \mathrm{~mm} \times 4 \mathrm{~mm} \times 3 \mathrm{~mm}$ centered on the VOI. The typical line width of water resonance (FWHM) was $8-10 \mathrm{~Hz}$. All spectra were acquired with a spectral width of $5 \mathrm{kHz}, 4096$ complex data points, mixing time of $20 \mathrm{~ms}$ and repetition time (TR) of $4 \mathrm{~s}$. Water spectrum was acquired as reference for quantification ( 4 averages) and then the metabolite spectrum was acquired using water suppression based on VAPOR, applied before the localized spectroscopy sequence. Spectra were acquired in 30 blocks of 12 averages stored separately on disk for a total acquisition time of $24 \mathrm{~min}$.

\subsubsection{Data analysis}

The 30 blocks were corrected for $B_{0}$ shift correction before summation and then an eddy-current correction was applied. Metabolite concentrations were quantified using LCModel as in previous studies (Tkac et al., 2003). This software is based on a frequency domain analysis, fitting the in vivo spectra with a linear combination of spectra of the individual metabolites (Provencher, 1993). The water signal was used as reference for quantification as in previous studies (Tkac et al., 2003) assuming a water concentration of $82.3 \%$ in the rat brain at PND20 (Tkac et al., 2003). The results provided the quantification of the following 17 metabolite concentrations: ascorbate (Asc), aspartate (Asp), creatine ( $\mathrm{Cr}$ ), $\gamma$-aminobutyric acid (GABA), glucose (Glc), glutamate (Glu), glutamine (Gln), glutathione (GSH), glycerophosphorylcholine (GPC), phosphorylcholine (PCho), myo-inositol (Ins), lactate (Lac), N-acetyl-aspartate (NAA), N-acetylaspartylglutamate (NAAG), phosphocreatine $(\mathrm{PCr})$, phosphorylethanolamine (PE), and taurine (Tau). The uncertainty on the quantification was estimated from the Cramér-Rao lower bounds (CRLBs) provided by the LCModel program. Only metabolites with a CRLB lower than $20 \%$ were considered in the statistical analysis.

Results are expressed as mean of means \pm SEM (histological parameters). Significant differences between controls and BPA-treated animals were assessed using a Mann-Whitney $U$ test for independent samples. A probability of $p<0.05$ was considered to be significant. Two animals were rejected from the MRS study (one in each group) based on poor spectral quality and health criteria.

\subsection{Histological analysis}

At PND20, pups from each group ( $n=7 \mathrm{BPA}$-treated animals, $n=4$ controls), taken from 2 to 4 different litters, were deeply anesthetized using ketalar $(50 \mathrm{mg} / \mathrm{ml}$; $0.2-0.5 \mathrm{ml}$, i.p.). Animals were perfused intracardially with $0.9 \% \mathrm{NaCl}$, then $4 \%$ paraformaldehyde. Brains were removed and postfixed in $4 \%$ paraformaldehyde overnight, then $20 \%$ in sucrose for $24 \mathrm{~h}$, and stored at $-80^{\circ} \mathrm{C}$ until sectioned. Coronal sections $(10 \mu \mathrm{m})$ at the level of the dorsal hippocampus were cut on a cryostat (Microm Cryo-Star HM 560M, Microm International, Germany). Three sections at $200 \mu \mathrm{m}$ intervals were collected from each animal and were alternately processed for haematoxylin and eosin $(\mathrm{H} \& \mathrm{E})$ staining and specific immunochemistry.

\subsubsection{Histology}

Sections were stained with $\mathrm{H} \& \mathrm{E}$ and assessed qualitatively for the presence of haemorrhages, lesions or infarcts.

\subsubsection{Immunohistochemistry}

The areal densities of astrocytes and neurons were assessed using glial fibrillary acidic protein (GFAP) and neuronal nuclei (NeuN), respectively. Non-specific binding was blocked by incubating sections in PBS containing $4 \%$ bovine serum albumin (BSA) for $30 \mathrm{~min}$ at room temperature. Sections were then incubated in rabbit anti-GFAP (1:400, Z334, Dako Cytomation, Denmark), and mouse anti-NeuN (1:400, MAB377, Chemicon, USA) in $0.25 \%$ BSA in PBS overnight at $4{ }^{\circ} \mathrm{C}$. This was followed with a $2 \mathrm{~h}$ incubation at room temperature with Alexa 555 anti-mouse (1:200) or Alexa 488 anti-rabbit (1:200) secondary antibodies, respectively (Molecular Probes, Invitrogen, Paisley, UK). Sections were rinsed in PBS and mounted using PBS-glycine media.

The extent of myelination was assessed using myelin basic protein (MBP). Activity of endogenous peroxidases was blocked with $0.3 \% \mathrm{H}_{2} \mathrm{O}_{2}$ in methanol for 20 min. Nonspecific binding was blocked by incubating the slides in $4 \%$ BSA for $30 \mathrm{~min}$ at room temperature. Sections were then incubated in primary antibody for $\operatorname{MBP}\left(1: 400\right.$, MAB386, Chemicon, USA) overnight at $4{ }^{\circ} \mathrm{C}$. This was followed by $60 \mathrm{~min}$ incubation at room temperature in secondary antibody (1:200 antimouse immunoglobulin G; Vectastain kit, Vector Laboratories, Burlingame, CA) and the avidin-biotin complex (1:200, Vector Laboratories). Sections were then developed with the chromagen, 3,3 diaminobenzidine (DAB) in $0.01 \%$ hydrogen peroxide.

\subsection{Quantitative analysis}

Quantitative analyses were performed using MetaMorph ${ }^{\circledR}$ Imaging System (Meta Imaging Software, Molecular Devices Corporation, PA, USA). Measurements were made on coded slides blinded to the observer with the codes not being disclosed until the conclusion of analyses.

\subsubsection{Areal density of neurons}

The density of NeuN-positive neurons was assessed in 6 fields within the motor and somatosensory cortices, respectively. The density of neurons in the hippocampus was also assessed ( 9 fields per animal). Values for each animal in each region were pooled and a mean value was calculated. A mean of means $\pm \mathrm{SEM}$ was calculated for each group.

\subsubsection{Areal density of astrocytes}

The density of GFAP-positive astrocytes was assessed in the cingulum. Six fields per animal were examined; values for each animal were pooled and a mean value was calculated. A mean of means \pm SEM was calculated for each group.

\subsubsection{Myelination}

The optical density (OD) of MBP-stained fibres was measured in the corpus callosum and cortex using Image (Rasband, 1997-2009). Eight fields within the corpus callosum, and six fields within the cortex, were examined. Optical density was measured at grey levels. Non-specific background ODs were measured at each brain level in a region devoid of MBP-immunostaining and were subtracted from the corpus callosum and cortex values. The area of MBP-positive fibres

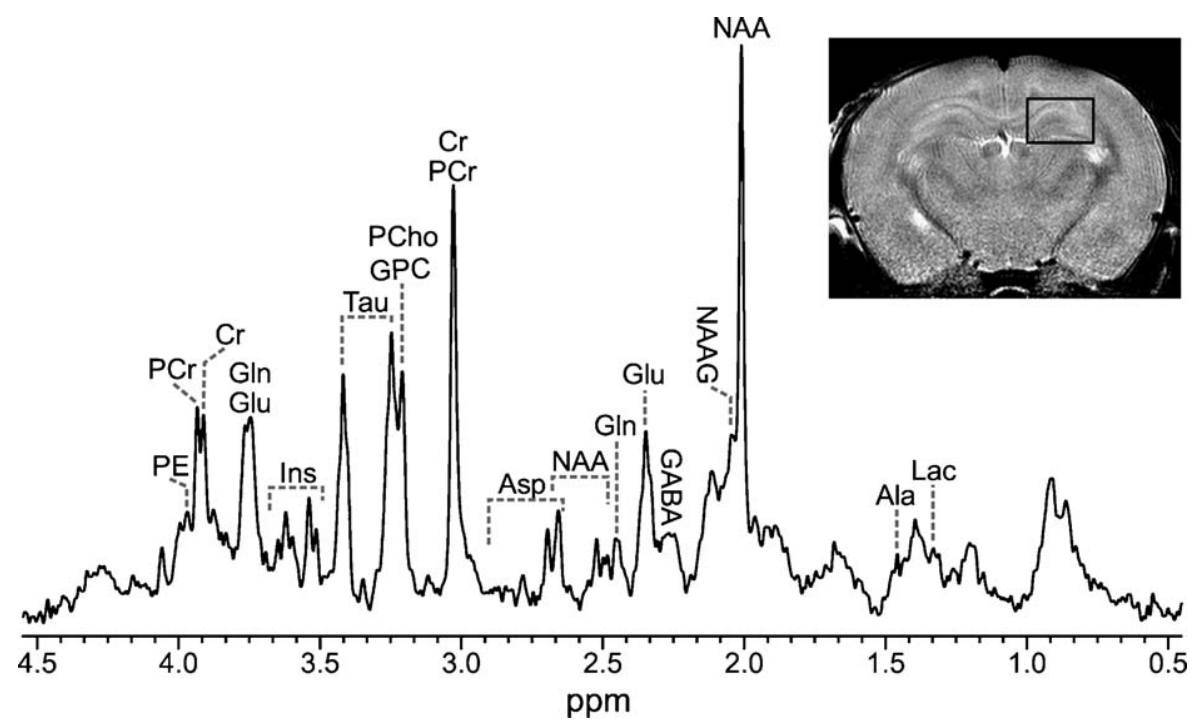

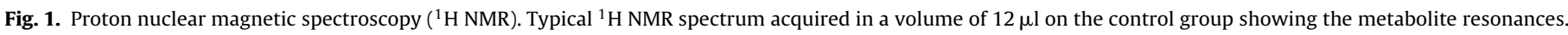

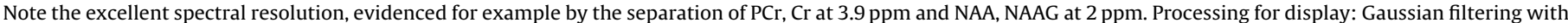

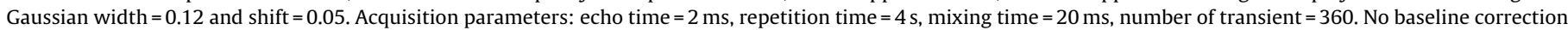

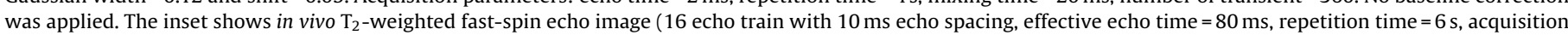
matrix $=256 \times 256, \mathrm{FOV}=20 \mathrm{~mm} \times 20 \mathrm{~mm}$, slice thickness $=1 \mathrm{~mm})$. The black box indicates the voxel position placed on the hippocampus $(3 \mathrm{~mm} \times 4 \mathrm{~mm} \times 3 \mathrm{~mm})$. 
in the cortex, relative to cortical size, was assessed using MetaMorph ${ }^{\circledR}$ Imaging System.

\section{Results}

\subsection{Nuclear magnetic resonance}

During the in vivo MRS measurements, the whole blood lactate measurement done before and after the experiment did not show any significant difference. Moreover, excellent spectral quality was achieved, as judged from water line width, ranging from $8 \mathrm{~Hz}$ to $10 \mathrm{~Hz}(9 \pm 0.7 \mathrm{~Hz})$ in the hippocampus (Fig. 1). Water suppression typically resulted in a residual water signal below that of N-acetyl-aspartate (NAA) and a highly stable baseline. Signal to noise ratio was on average $20 \pm 3$. In addition, the separation of NAAG from NAA and PCr from $\mathrm{Cr}$ was consistently observed (Fig. 1). Such high spectral quality allowed the quantification of the concentration of 21 metabolites (neurochemical profile), of which 14 had a precision better than $20 \%$ and especially $\mathrm{Cr}$, Tau, $\mathrm{Cr}+\mathrm{PCr}$, Glu, NAA, NAA+ NAAG had a precision better than $5 \%$ (Table 2). Only these 14 metabolites were analysed for the study.

Overall, the neurochemical profile of the BPA-treated animals was very similar to that of the control group (Table 3 ). However, a statistically significant increase of glutamate (Glu) from $[\mathrm{Glu}]_{\text {control }}=9.0 \pm 0.3 \mu \mathrm{mol} / \mathrm{g}$, to $[\mathrm{Glu}]_{\mathrm{BPA}}=9.7 \pm 0.2 \mu \mathrm{mol} / \mathrm{g}$ $(p=0.035$, unpaired $t$-test) was noted. Interestingly, aspartate (Asp), which is metabolically linked to Glu by the transaminase reaction showed a trend towards a decrease. Consequently, the Glu/Asp ratio significantly increased from $[\mathrm{Glu} / \text { Asp }]_{\text {control }}=4 \pm 0.3 \mu \mathrm{mol} / \mathrm{g}$ to $[\mathrm{Glu} / \mathrm{Asp}]_{\mathrm{BPA}}=5.5 \pm 0.4 \mu \mathrm{mol} / \mathrm{g}$ $(p=0.02)$ (Fig. 2), whereas the sum of Asp + Glu concentrations did not change significantly $(p>0.5)$. Glu and Asp resonances were well separated in the ${ }^{1} \mathrm{H}$ spectra, therefore the correlation between these two metabolites during the quantification was very low (average correlation coefficient of 0.04). However, it is important to notice that Asp concentration was quantified with a CRLB of $35 \%$ in the BPA-treated animals. Due to the biological implication of Asp in this model, its quantification was kept as a significant result. Furthermore, the Glu/Asp ratio was still significant despite the higher variability on the Asp concentration.

Table 2

Cramér-Rao lower bound (CRLB) of the metabolite concentration quantified with LCModel.

\begin{tabular}{lcc}
\hline Metabolite & CRLB [\%] & \\
\cline { 2 - 3 } & Control & BPA \\
\hline Asc & 10 & 11 \\
Asp & 19 & 35 \\
Cr & 7 & 7 \\
PCr & 5 & 5 \\
GABA & 12 & 12 \\
Glc & 13 & 15 \\
Gln & 13 & 13 \\
Glu & 4 & 3 \\
GSH & 18 & 19 \\
Ins & 6 & 6 \\
Lac & 25 & 24 \\
NAA & 2 & 2 \\
NAAG & 19 & 21 \\
PE & 8 & 9 \\
Tau & 3 & 3 \\
NAA + NAAG & 2 & 2 \\
Glu + Gln & 4 & 3 \\
GPC + PCho & 9 & 8 \\
Cr+ PCr & 2 & 2 \\
All & & 3
\end{tabular}

All selected metabolites have a CRLB lower than $20 \%$ assuring a robust quantification of metabolite concentrations.
Table 3

Metabolite concentration.

\begin{tabular}{|c|c|c|}
\hline \multirow[t]{2}{*}{ Metabolite } & \multicolumn{2}{|c|}{ Concentration $[\mu \mathrm{mol} / \mathrm{g}] \pm \mathrm{SE}$} \\
\hline & Control & BPA \\
\hline Asc & $3.3 \pm 0.1$ & $3.2 \pm 0.1$ \\
\hline Asp & $2.3 \pm 0.2$ & $1.9 \pm 0.2$ \\
\hline $\mathrm{Cr}$ & $3.8 \pm 0.1$ & $3.8 \pm 0.1$ \\
\hline $\mathrm{PCr}$ & $5.2 \pm 0.1$ & $5.3 \pm 0.1$ \\
\hline GABA & $1.9 \pm 0.1$ & $1.9 \pm 0.1$ \\
\hline Glc & $3.4 \pm 0.2$ & $3.2 \pm 0.1$ \\
\hline Gln & $2.0 \pm 0.2$ & $1.9 \pm 0.1$ \\
\hline Glu & $9.0 \pm 0.2$ & $9.7 \pm 0.2^{*}$ \\
\hline GSH & $1.0 \pm 0.1$ & $0.9 \pm 0.1$ \\
\hline Ins & $4.0 \pm 0.1$ & $4.1 \pm 0.2$ \\
\hline Lac & $0.7 \pm 0.1$ & $0.9 \pm 0.2$ \\
\hline NAA & $10.0 \pm 0.2$ & $9.6 \pm 0.2$ \\
\hline NAAG & $0.9 \pm 0.1$ & $0.8 \pm 0.1$ \\
\hline PE & $3.1 \pm 0.1$ & $3.3 \pm 0.1$ \\
\hline Tau & $12.0 \pm 0.3$ & $12.00 \pm 0.1$ \\
\hline $\mathrm{NAA}+\mathrm{NAAG}$ & $10.8 \pm 0.1$ & $10.44 \pm 0.2$ \\
\hline Glu + Gln & $11.0 \pm 0.4$ & $11.59 \pm 0.2$ \\
\hline GPC + PCho & $1.0 \pm 0.1$ & $1.1 \pm 0.1$ \\
\hline $\mathrm{Cr}+\mathrm{PCr}$ & $8.9 \pm 0.2$ & $9.2 \pm 0.2$ \\
\hline Glu + Asp & $11.2 \pm 0.9$ & $11.57 \pm 0.5$ \\
\hline $\mathrm{PCr} / \mathrm{Cr}$ & $1.4 \pm 0.1$ & $1.4 \pm 0.1$ \\
\hline Glu/Gln & $4.6 \pm 0.3$ & $5.3 \pm 0.2$ \\
\hline Glu/Asp & $4.0 \pm 0.3$ & $5.5 \pm 0.4^{*}$ \\
\hline
\end{tabular}

Concentrations of brain metabolites present in the hippocampus at PND20 in the control and BPA groups (mean \pm SEM).

${ }^{*} p<0.05$, versus control offspring.

\subsection{Histological analysis}

There were no gross morphologic changes (haemorrhages, lesions or infarcts) in the brains of either control or BPA-treated offspring.

The morphology and density of NeuN-positive neurons were assessed in the motor and somatosensory cortices, and hippocampus. In both the motor and somatosensory cortices, no striking changes in neuronal morphology could be seen and there was no significant difference between control and BPA-treated offspring in the density of neurons ( $p>0.05$, Table 4$)$. In the hippocampus, neuronal morphology appeared to be similar in the two groups but the NeuN-positive neuron density was decreased in the BPA-treated offspring when compared to controls $(p<0.05)$.

The density of GFAP-positive cells was assessed in the cingulum. Astrocytes appeared with increased processes in the BPA treated pups compared to controls. GFAP density in astrocytes was increased in BPA-treated offspring compared to controls $(p<0.05$; Table 4).

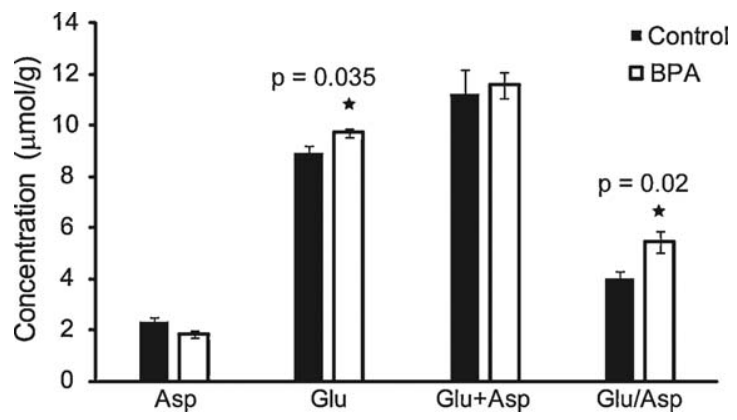

Fig. 2. ${ }^{1} \mathrm{H}$ NMR metabolite concentrations. Concentration of glutamate (Glu) and aspartate (Asp) in hippocampus at PND20 in the control group (black) and in the BPA group (white). Glu was significantly increased in the BPA group $(p=0.035)$. The Glu/Asp ratio shows a significant decrease $(p=0.02)$. Error bars are the error of the mean. ${ }^{*} p<0.05$, versus control offspring. 
Table 4

Histology results.

\begin{tabular}{lcc}
\hline & Control & BPA \\
\hline Motor cortex (neurons $/ \mathrm{mm}^{2}$ ) & $1281.0 \pm 72.0$ & $1162.9 \pm 44.1$ \\
Somatosensory cortex $\left(\right.$ neurons $/ \mathrm{mm}^{2}$ ) & $888.7 \pm 44.1$ & $882.3 \pm 45.1$ \\
Hippocampus (neurons $/ \mathrm{mm}^{2}$ ) & $4437.6 \pm 136.0$ & $3753.0 \pm 83.0^{*}$ \\
Astrocyte density (GFAP $/ \mathrm{mm}^{2}$ ) & $664.6 \pm 102.7$ & $1080.6 \pm 104.3^{*}$ \\
Myelin: cortex (OD) & $0.135 \pm 0.014$ & $0.125 \pm 0.004$ \\
Myelin: corpus callosum (OD) & $0.291 \pm 0.026$ & $0.317 \pm 0.017$ \\
Percent of myelination fibres in cortex (\%) & $53.1 \pm 1.4$ & $56.2 \pm 0.7$ \\
\hline
\end{tabular}

Density of neurons and glia in the cortex and hippocampus of control and BPAtreated offspring.

$p<0.05$, versus control offspring.

In order to determine the extent of myelination, MBP staining in the corpus callosum and cortex was assessed. There was no difference in MBP staining in the corpus callosum or cortex $(p>0.05$; Table 4) between the groups. In addition, the area of MBP-positive fibres in the cortex, relative to cortical area, was not significantly different between the groups ( $p>0.05)$.

\section{Discussion}

In the present study, a combination of ${ }^{1} \mathrm{H}$ MRS and histopathology was used in order to characterize different mechanisms likely to contribute to adverse neurodevelopmental outcomes following BPA exposure. This study has shown that offspring from dams exposed to BPA during pregnancy and lactation, exhibit alterations in localized cerebral metabolism, in neuronal and glial development. The NMR in vivo results show that a consistent neurochemical profile can be obtained from small volumes in the developing rat hippocampus, with a precision of better than $20 \%$ allowing the investigation of the neurochemical profile sequelae of environmental disruptors on brain development. Treatment of rat pups with BPA altered the Glu/Asp ratio reinforcing the determination of the neurochemical profile as a robust investigational tool in environmental science.

The stable concentration of whole blood lactate during the ${ }^{1} \mathrm{H}$ MRS confirms the non-invasive capability of the MRS techniques.

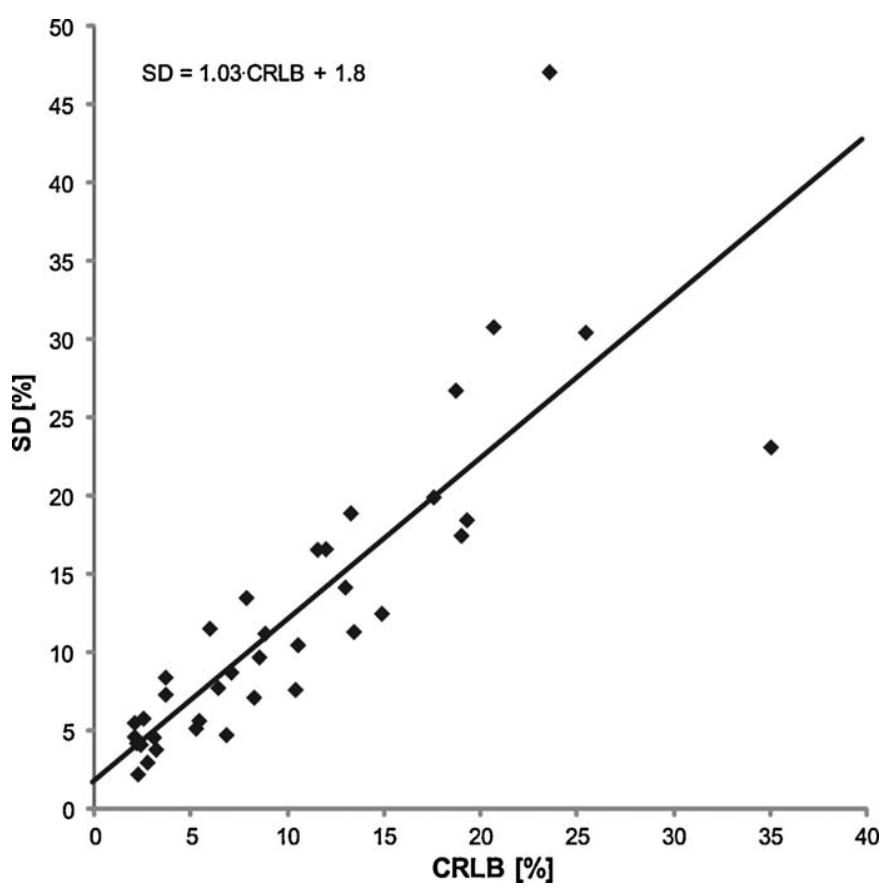

Fig. 3. Correlation graph between SD and CRLB. The CRLB follows very closely to the standard deviation within the two groups with a correlation factor close to 1 .
The neurochemical profile and average precision of $12 \%$ of the control group was in excellent agreement with a previous study on developing rat brain (Tkac et al., 2003). The precision of the measurement was determined from the CRLB, a standard output of the LCModel program. The CRLB follows very closely the SD inside the group with a correlation factor close to one (Fig. 3). Consequently this implies negligible inter-animal variability.

BPA pre and post-natal treatments resulted in small changes in the neurochemical profile that reached statistical significance for glutamate, where an increase was noted. This Glu increase could be reflecting an alteration of Glu uptake by the astrocytes through ERs (Sato et al., 2003). We further observed a trend for Asp to decrease and thus evaluated the Glu/Asp ratio, which was significantly increased. However, it is important to notice that Asp concentration was quantified with a CRLB of only $35 \%$ in the BPAtreated animals, which may explain why Asp decrease was not significant. Glu and Asp fulfil many roles in mammalian cells of the nervous system and are metabolically linked by the transaminase reaction. The altered Glu/Asp ratio may thus indicate an impaired aspartate amino-transferase reaction, a key step in the malate-aspartate shuttle, a scheme of which is shown in Fig. 4. The malate-aspartate shuttle serves to transport reducing equivalents across the inner mitochondrial membrane and is thus a crucial component in glucose respiration, which powers almost all energy demands in the brain. The malate-aspartate shuttle has a rate-controlling step at the Glu/Asp antiporter, which transports a proton and glutamate in exchange for Asp across the inner mitochondrial membrane. The observation of increased Glu is consistent with excess glutamate that is not adequately transported into the mitochondrion. Likewise, the reduced Asp is consistent with insufficient transport of Asp out of the mitochondrion, compared to the activity of the cytosolic dehydrogenases. It is thus likely that the altered Asp/Glu ratio indicates impaired malate-aspartate shuttle activity and thus implies impaired mitochondrial function.

In this context, it is of interest to note that BPA has been reported to interfere with mitochondrial integrity. Nakagawa and Tayama (2000) demonstrated in isolated rat's liver mitochondria that the intracellular levels of ATP are decreased due to an inhibition of $\mathrm{NAD}^{+}$- and FAD-linked respiration. The malate-aspartate shuttle is central to $\mathrm{NAD}^{+}$regeneration. Bindhumol et al. (2003) have also reported a decrease in the activity of antioxidant enzymes, which further support impaired mitochondrial function. Furthermore, it has been shown that BPA induced ROS production and oxidative stress, which compromised mitochondrial function (Ooe et al., 2005).

In vivo, BPA exposure during pregnancy and lactation has been shown to decrease neuronal density in the hippocampus (Choi et al., 2007; Ogiue-Ikeda et al., 2008; Sato et al., 2002). Several studies have reported alterations in hippocampal morphology with altered estradiol and testosterone induced spine synapse formation not only in rats but also in non human primates following BPA exposure (Lee et al., 2008; Leranth et al., 2008; Maclusky et al., 2005; Zhou et al., 2009). Synapse plasticity in the striatum is also perturbed after pre- and postnatal exposures with alteration of the function of dopaminergic receptors and disturbance in the developmental pattern of synaptic plasticity, which causes controlling deficits in motor behaviour (Zhou et al., 2009). Earlier studies (Ikemoto et al., 2004) reported that the exposure to BPA during pre- and postnatal developments has long-lasting effects on central dopaminergic systems linked with behavioural rewarding effects. In vitro exposure to BPA results in a marked influence on synaptogenesis and potentially neuronal vulnerability in hippocampal cultures (Sato et al., 2002; Zhou et al., 2009). The effects of BPA on synaptogenesis could be mediated through several mechanisms, including a number of receptor systems, such as estrogen (ER- $\alpha$ and ER- $\beta$ ) and thyroid receptors (TRs). As synapse remodelling in the 


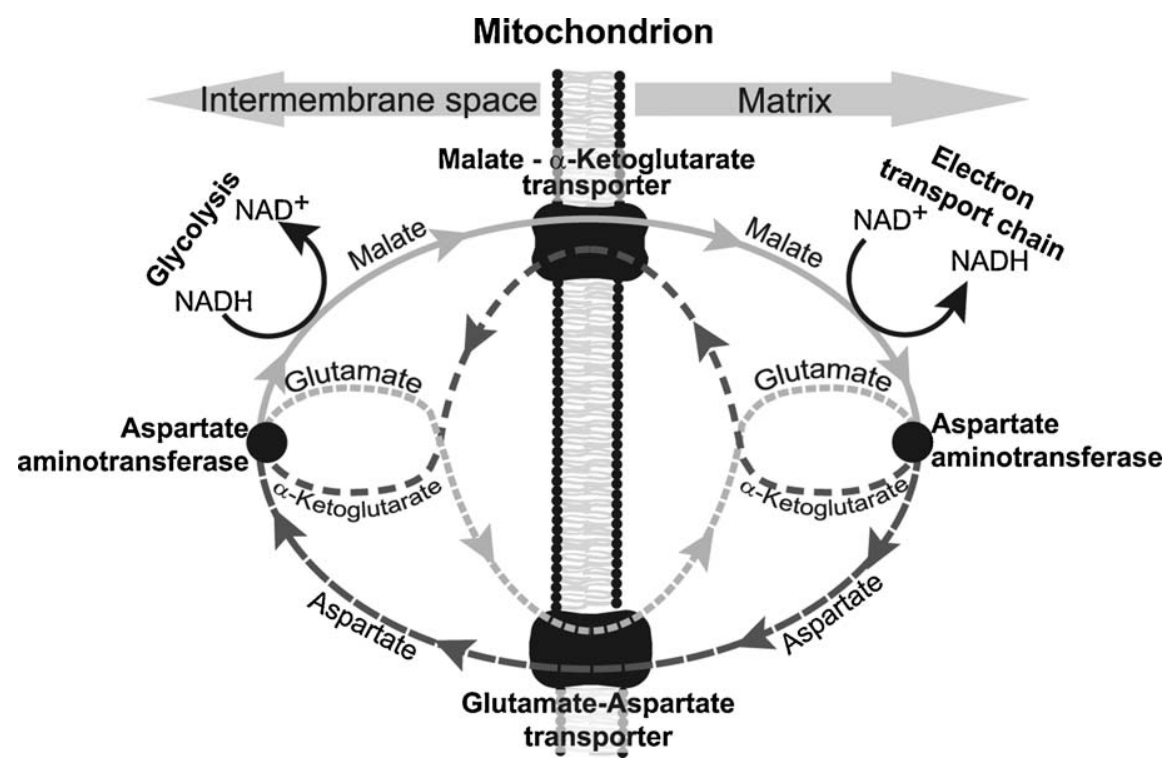

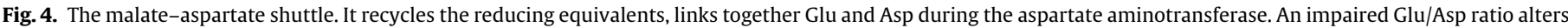
the malate-aspartate shuttle function.

hippocampus is implicated in memory acquisition, retention and learning (Silva, 2003), it is likely that BPA exposure during critical periods of development could lead to altered cognitive function.

Further, direct neuronal toxicity of BPA induced apoptotic cell death have been suggested to be through calcium, ROS, ERK, and JNK in vitro. In contrast, NF-kappaB cascade was activated for survival signalling after BPA treatment (Lee et al., 2008). Therefore the mechanisms leading to decrease of hippocampal volume and neuronal density seen in our study are likely multi-factorial not only with a direct toxicity of BPA on the developing neurons, but also with BPA's ability to alter synaptogenesis that would lead to increased developmental pruning of neurons unable to establish sufficient synaptic activity for survival.

The decrease in hippocampal neuronal density and volume may reflect inadequate accelerated neuronal differentiation and maturation with an increase in the normal developmental process of programmed cell death. On the other hand, BPA exposure has also been shown to increase cell growth of immature neurons and neurospheres in vitro (Kubo et al., 2004), and in vivo, to promote Purkinje dendritic growth (Shikimi et al., 2004) and accelerate neocortical neuronal differentiation/migration (Nakamura et al., 2006). This neuronal differentiation/migration could be transitory and counter-balanced by the altered synaptogenesis and increased neuronal pruning. The current study cannot differentiate between these mechanisms of altered neuronal development but certainly confirms that gestational and postnatal exposition to BPA provides altered hippocampal development.

The areal density of GFAP-positive astrocytes was increased in BPA-treated offspring compared to controls (Cai et al., 2006). Several mechanisms of astrocyte activation have been linked to BPA exposure. Hydroxy radical formation has been shown to be increased in adult mouse brain following 5 days of BPA exposure (Kabuto et al., 2003) and BPA exposure during pregnancy and lactation increases lipid peroxidation in the postnatal mice brain (Kabuto et al., 2004). Thus, the activation of astrocytes in the white matter observed in the present study may be due to BPA-induced tissue oxidative stress and peroxidation. In vitro, low-level BPA has been shown to increase the production of GFAP protein in differentiating astrocyte progenitor cells, an effect mediated through the activation of signal transducer and activator of transcription 3 (STAT3), and mothers against decapentaplegic homolog 1 (Smad1) (Yamaguchi et al., 2006). Several recent reports show that BPA binds to thyroid hormone receptors (THRs), and has selective effects on thyroid function (Bindhumol et al., 2003; Zoeller, 2007). Thyroid hormone $(\mathrm{TH})$ is a pivotal factor in astrocyte development (Adachi et al., 2002; Seiwa et al., 2004), thus, the increase in GFAP expression in the present study may reflect BPA's ability to influence TH signalling in the developing brain. The effects of BPA on brain development are also mediated for the most part by ERs and astrocytes are important target cells for estrogens and express all types of ERs during development and in the adult brain (Chaban et al., 2004; Hosli et al., 2001; Sato et al., 2003). Treatment of mouse purified astrocytes with BPA in vitro causes astrocyte activation, as detected by an increase in the levels of GFAP-immunoreactivity (Miyatake et al., 2006). Thus, GFAP upregulation and increase in astrocyte observed in the present study may also be ER-mediated. Further, estrogens and BPA down-regulate L-Glu uptake activity of astrocytes through ERs, which regulates the L-Glu concentration in the synaptic clefts, thereby altering synaptic transmissions and glutamate excitotoxicity. BPA might then alter not only directly synapse formation and activity but also through ERs on astrocytes (Sato et al., 2003). In summary, BPA has the capability of modulating astrocyte development and function through several different mechanisms leading to changes in the glial role and function within the brain.

Taking into account the significant change in the Glu/Asp ratio in the hippocampus observed by MRS, it is possible that the decrease in neuronal density in the hippocampus may be secondary to impaired mitochondrial function and reduced ability of the brain to oxidize glucose, thus leading to cell death. A critical experiment for future studies will be to examine the long-term effects of BPAexposure on the brain, as alterations in brain structure may appear secondary to further impairments in cerebral metabolism, which may exacerbate during normal aging.

In conclusion, exposure to low dose BPA during gestation and lactation results in subtle and regional neuronal and glial alterations in brain development in offspring. Furthermore, BPA exposure leads to significant changes in the Glu/Asp ratio in the hippocampus, which is postulated to reflect impaired mitochondrial function and probably implicates a reduced ability of the brain to oxidize glucose especially in conditions of elevated energetic demand. The changes we observed in brain structure and metabolism after prenatal BPA exposure will likely imply longlasting effects on cognitive development and function. 


\section{Acknowledgments}

This study was supported by Centre d'Imagerie BioMédicale (CIBM) of the UNIL, UNIGE, HUG, CHUV, EPFL, the Leenaards and Jeantet Foundations and the FNS Grant PNR 50 SNF 405040-108'713 (MA/PSH).

\section{References}

Adachi, T., Takanaga, H., Sakurai, Y., Ishido, M., Kunimoto, M., Asou, H., 2002. Influence of cell density and thyroid hormone on glial cell development in primary cultures of embryonic rat cerebral hemisphere. J. Neurosci. Res. 69, 61-71.

Aydogan, M., Korkmaz, A., Barlas, N., Kolankaya, D., 2008. The effect of vitamin C on bisphenol A, nonylphenol and octylphenol induced brain damages of male rats. Toxicology 249, 35-39.

Bindhumol, V., Chitra, K.C., Mathur, P.P., 2003. Bisphenol A induces reactive oxygen species generation in the liver of male rats. Toxicology 188, 117-124.

Cai, Z., Lin, S., Fan, L.W., Pang, Y., Rhodes, P.G., 2006. Minocycline alleviates hypoxic-ischemic injury to developing oligodendrocytes in the neonatal rat brain. Neuroscience 137, 425-435.

Calafat, A.M., Kuklenyik, Z., Reidy, J.A., Caudill, S.P., Ekong, J., Needham, L.L., 2005. Urinary concentrations of bisphenol A and 4-nonylphenol in a human reference population. Environ. Health Perspect. 113, 391-395.

Chaban, V.V., Lakhter, A.J., Micevych, P., 2004. A membrane estrogen receptor mediates intracellular calcium release in astrocytes. Endocrinology 145, 3788-3795.

Choi, I.S., Cho, J.H., Park, E.J., Park, J.W., Kim, S.H., Lee, M.G., Choi, B.J., Jang, I.S., 2007. Multiple effects of bisphenol A, an endocrine disrupter, on GABA(A) receptors in acutely dissociated rat CA3 pyramidal neurons. Neurosci. Res. 59, 8-17.

Dessi-Fulgheri, F., Porrini, S., Farabollini, F., 2002. Effects of perinatal exposure to bisphenol A on play behavior of female and male juvenile rats. Environ. Health Perspect. 110 (Suppl. 3), 403-407.

Facciolo, R.M., Alo, R., Madeo, M., Canonaco, M., Dessi-Fulgheri, F., 2002. Early cerebral activities of the environmental estrogen bisphenol A appear to act via the somatostatin receptor subtype sst(2). Environ. Health Perspect. 110 (Suppl. 3), 397-402.

Farabollini, F., Porrini, S., Della Seta, D., Bianchi, F., Dessi-Fulgheri, F., 2002. Effects of perinatal exposure to bisphenol A on sociosexual behavior of female and male rats. Environ. Health Perspect. 110 (Suppl. 3), 409-414.

Fujimaki, K., Arakawa, C., Yoshinaga, J. Watanabe, C., Serizawa, S., Imai, H., Shiraishi, H., Mizumoto, Y., 2004. Estimation of intake level of bisphenol A in Japanese pregnant women based on measurement of urinary excretion level of the metabolite. Nippon Eiseigaku Zasshi 59, 403-408.

Gruetter, R., 1993. Automatic, localized in vivo adjustment of all first- and secondorder shim coils. Magn. Reson. Med. 29, 804-811.

Hosli, E., Jurasin, K., Ruhl, W., Luthy, R., Hosli, L., 2001. Colocalization of androgen, estrogen and cholinergic receptors on cultured astrocytes of rat central nervous system. Int. J. Dev. Neurosci. 19, 11-19.

Ikemoto, S., Witkin, B., Zangen, A., Wise, R., 2004. Rewarding Effects of AMPA Administration into the Supramammillary or Posterior Hypothalamic Nuclei But Not the Ventral Tegmental Area. J. Neurosci. 24, 5758-5765.

Ikezuki, Y., Tsutsumi, O., Takai, Y., Kamei, Y., Taketani, Y., 2002. Determination of bisphenol A concentrations in human biological fluids reveals significant early prenatal exposure. Hum. Reprod. 17, 2839-2841.

Ishido, M., Masuo, Y., Kunimoto, M., Oka, S., Morita, M., 2004. Bisphenol A causes hyperactivity in the rat concomitantly with impairment of tyrosine hydroxylase immunoreactivity. J. Neurosci. Res. 76, 423-433.

Kabuto, H., Amakawa, M., Shishibori, T., 2004. Exposure to bisphenol A during embryonic/fetal life and infancy increases oxidative injury and causes underdevelopment of the brain and testis in mice. Life Sci. 74, 2931-2940.

Kabuto, H., Hasuike, S., Minagawa, N., Shishibori, T., 2003. Effects of bisphenol A on the metabolisms of active oxygen species in mouse tissues. Environ. Res. 93, 31-35.

Kubo, T., Maezawa, N., Osada, M., Katsumura, S., Funae, Y., Imaoka, S., 2004. Bisphenol $A$, an environmental endocrine-disrupting chemical, inhibits hypoxic response via degradation of hypoxia-inducible factor 1alpha (HIF-1alpha): structural requirement of bisphenol A for degradation of HIF-1alpha. Biochem. Biophys. Res. Commun. 318, 1006-1011.

Lee, S., Suk, K., Kim, I.K., Jang, I.S., Park, J.W., Johnson, V.J., Kwon, T.K., Choi, B.J., Kim, S.H., 2008. Signaling pathways of bisphenol A-induced apoptosis in hippocampal neuronal cells: role of calcium-induced reactive oxygen species, mitogen-activated protein kinases, and nuclear factor-kappaB. J. Neurosci. Res. $86,2932-2942$

Leranth, C., Hajszan, T., Szigeti-Buck, K., Bober, J., Maclusky, N.J., 2008. Bisphenol A prevents the synaptogenic response to estradiol in hippocampus and prefrontal cortex of ovariectomized nonhuman primates. Proc. Natl. Acad. Sci. U.S.A. 105, 14187-14191.

Maclusky, N.J., Hajszan, T., Leranth, C., 2005. The environmental estrogen bisphenol a inhibits estradiol-induced hippocampal synaptogenesis. Environ. Health Perspect. 113, 675-679.

Miyatake, M., Miyagawa, K., Mizuo, K., Narita, M., Suzuki, T., 2006. Dynamic changes in dopaminergic neurotransmission induced by a low concentration of bisphenol-A in neurones and astrocytes. J. Neuroendocrinol. 18, 434-444.

Nakagawa, Y., Tayama, S., 2000. Metabolism and cytotoxicity of bisphenol A and other bisphenols in isolated rat hepatocytes. Arch. Toxicol. 74, 99-105.
Nakamura, K., Itoh, K., Yaoi, T., Fujiwara, Y., Sugimoto, T., Fushiki, S., 2006. Murine neocortical histogenesis is perturbed by prenatal exposure to low doses of bisphenol A. J. Neurosci. Res. 84, 1197-1205.

Ogiue-Ikeda, M. Tanabe, N., Mukai, H., Hojo, Y., Murakami, G., Tsurugizawa, T. Takata, N., Kimoto, T., Kawato, S., 2008. Rapid modulation of synaptic plasticity by estrogens as well as endocrine disrupters in hippocampal neurons. Brain Res. Rev. 57, 363-375.

Ooe, H., Taira, T., Iguchi-Ariga, S.M., Ariga, H., 2005. Induction of reactive oxygen species by bisphenol A and abrogation of bisphenol A-induced cell injury by DJ-1. Toxicol. Sci. 88, 114-126.

Palanza, P., Gioiosa, L., Vom Saal, F.S., Parmigiani, S., 2008. Effects of developmental exposure to bisphenol A on brain and behavior in mice. Environ. Res. 108, $150-157$.

Paris, F., Balaguer, P., Terouanne, B., Servant, N., Lacoste, C., Cravedi, J.P., Nicolas, J.C. Sultan, C., 2002. Phenylphenols, biphenols, bisphenol-A and 4-tert-octylphenol exhibit alpha and beta estrogen activities and antiandrogen activity in reporter cell lines. Mol. Cell. Endocrinol. 193, 43-49.

Pfeuffer, J., Tkac, I., Provencher, S.W., Gruetter, R., 1999. Toward an in vivo neurochemical profile: quantification of 18 metabolites in short-echo-time (1)H NMR spectra of the rat brain. J. Magn. Reson. 141, 104-120.

Provencher, S.W., 1993. Estimation of metabolite concentrations from localized in vivo proton NMR spectra. Magn. Reson. Med. 30, 672-679.

Raman, L., Tkac, I., Ennis, K., Georgieff, M.K., Gruetter, R., Rao, R., 2005. In vivo effect of chronic hypoxia on the neurochemical profile of the developing rat hippocampus. Brain Res. Dev. Brain Res. 156, 202-209.

Ranjit, N., Siefert, K., Padmanabhan, V., 2009. Bisphenol-A and disparities in birth outcomes: a review and directions for future research. J. Perinatol. 30, 2-9.

Rao, R., Tkac, I., Townsend, E.L., Gruetter, R., Georgieff, M.K., 2003. Perinatal iron deficiency alters the neurochemical profile of the developing rat hippocampus. J. Nutr. 133, 3215-3221.

Rasband, W.S., 1997-2009. ImageJ, Bethesda, MD, USA, http://rsb.info.nih.gov/ij/.

Rubin, B.S., Murray, M.K., Damassa, D.A., King, J.C., Soto, A.M., 2001. Perinatal exposure to low doses of bisphenol A affects body weight, patterns of estrous cyclicity, and plasma LH levels. Environ. Health Perspect. 109, 675-680.

Sato, K., Matsuki, N., Ohno, Y., Nakazawa, K., 2002. Effects of 17beta-estradiol and xenoestrogens on the neuronal survival in an organotypic hippocampal culture. Neuroendocrinology 76, 223-234.

Sato, K., Matsuki, N., Ohno, Y., Nakazawa, K., 2003. Estrogens inhibit L-glutamate uptake activity of astrocytes via membrane estrogen receptor alpha. J. Neurochem. 86, 1498-1505.

Schonfelder, G., Wittfoht, W., Hopp, H., Talsness, C.E., Paul, M., Chahoud, I., 2002. Parent bisphenol A accumulation in the human maternal-fetal-placental unit. Environ. Health Perspect. 110, A703-A707.

Seiwa, C., Nakahara, J., Komiyama, T., Katsu, Y., Iguchi, T., Asou, H., 2004. Bisphenol A exerts thyroid-hormone-like effects on mouse oligodendrocyte precursor cells. Neuroendocrinology 80, 21-30.

Shikimi, H., Sakamoto, H., Mezaki, Y., Ukena, K., Tsutsui, K., 2004. Dendritic growth in response to environmental estrogens in the developing Purkinje cell in rats. Neurosci. Lett. 364, 114-118.

Silva, A.J., 2003. Molecular and cellular cognitive studies of the role of synaptic plasticity in memory. J. Neurobiol. 54, 224-237.

Somm, E., Schwitzgebel, V.M., Toulotte, A., Cederroth, C.R., Combescure, C., Nef, S. Aubert, M.L., Huppi, P.S., 2009. Perinatal exposure to bisphenol a alters early adipogenesis in the rat. Environ. Health Perspect. 117, 1549-1555.

Takeuchi, T., Tsutsumi, O., 2002. Serum bisphenol a concentrations showed gender differences, possibly linked to androgen levels. Biochem. Biophys. Res. Commun. 291, 76-78.

Tkac, I., Rao, R., Georgieff, M.K., Gruetter, R., 2003. Developmental and regional changes in the neurochemical profile of the rat brain determined by in vivo ${ }^{1} \mathrm{H}$ NMR spectroscopy. Magn. Reson. Med. 50, 24-32.

Tkac, I., Starcuk, Z., Choi, I.Y., Gruetter, R., 1999. In vivo ${ }^{1} \mathrm{H}$ NMR spectroscopy of rat brain at $1 \mathrm{~ms}$ echo time. Magn. Reson. Med. 41, 649-656.

Vandenberg, L.N., Maffini, M.V., Sonnenschein, C., Rubin, B.S., Soto, A.M., 2009 Bisphenol-A and the great divide: a review of controversies in the field of endocrine disruption. Endocr. Rev. 30, 75-95.

Vom Saal, F.S., Cooke, P.S., Buchanan, D.L., Palanza, P., Thayer, K.A., Nagel, S.C., Parmigiani, S., Welshons, W.V., 1998. A physiologically based approach to the study of bisphenol A and other estrogenic chemicals on the size of reproductive organs, daily sperm production, and behavior. Toxicol. Ind. Health 14, 239-260.

Xing, L., Xu, Y., Xiao, Y., Shang, L., Liu, R., Wei, X., Jiang, J., Hao, W., 2010. Embryotoxic and teratogenic effects of the combination of bisphenol A and genistein on in vitro cultured postimplantation rat embryos. Toxicol. Sci. 115, 577-588.

Yamaguchi, H., Zhu, J., Yu, T., Sasaki, K., Umetsu, H., Kidachi, Y., Ryoyama, K., 2006. Low-level bisphenol A increases production of glial fibrillary acidic protein in differentiating astrocyte progenitor cells through excessive STAT3 and Smad1 activation. Toxicology 226, 131-142.

Ye, X., Kuklenyik, Z., Needham, L.L., Calafat, A.M., 2005. Quantification of urinary conjugates of bisphenol A, 2,5-dichlorophenol, and 2-hydroxy-4methoxybenzophenone in humans by online solid phase extraction-high performance liquid chromatography-tandem mass spectrometry. Anal. Bioanal. Chem. 383, 638-644.

Zhou, R., Zhang, Z., Zhu, Y., Chen, L., Sokabe, M., Chen, L., 2009. Deficits in development of synaptic plasticity in rat dorsal striatum following prenatal and neonatal exposure to low-dose bisphenol A. Neuroscience 159, 161-171.

Zoeller, R.T., 2007. Environmental chemicals impacting the thyroid: targets and consequences. Thyroid 17, 811-817. 